\title{
Los médicos especialistas en México
}

\author{
Gerhard Heinze-Martin, Víctor Hugo Olmedo-Canchola, Germán Bazán-Miranda, \\ Napoléon Andrés Bernard-Fuentes y Diana Patricia Guízar-Sánchez \\ Universidad Nacional Autónoma de México, División de Estudios de Posgrado, Facultad de Medicina, Ciudad de México, México
}

\section{Resumen}

Introducción: No hay datos recientes en México sobre el estado de los médicos especialistas que permitan diseñar políticas de formación y empleo de los recursos humanos para la salud. Objetivo: Analizar el estado actual de los médicos especialistas en México: número, distribución (geográfica, por sexo y especialidad), vigencia de la certificación (por sexo y especialidad) y tasa de especialistas por 100000 habitantes. Método: estudio transversal descriptivo. Se consultaron múltiples fuentes disponibles en México: consejos, asociaciones, colegios, instituciones hospitalarias, universidades y otros. Resultados: Se contabilizaron 147910 especialistas para una población de 123518272 habitantes; 69 \% contaba con certificación vigente de la especialidad. Se obtuvo una tasa de 119 especialistas por 100000 habitantes; 54.2 \% se encontraba en la Ciudad de México, Estado México, Jalisco y Nuevo León. En promedio había 1.7 especialistas varones por cada mujer. Conclusiones: El número de especialistas es inferior al recomendado internacionalmente e insuficiente para cubrir las necesidades en salud del país. La distribución por género está cambiando hacia una más equitativa. Los especialistas están agrupados mayormente en zonas urbanas. Es el primer censo de especialistas con base en el número comprobable de médicos, que permitirá el diseño de políticas de planificación de recursos humanos en salud.

PALABRAS CLAVE: Médicos especialistas. Recursos humanos en salud. Especialistas certificados. México.

\begin{abstract}
Introduction: There are no recent data in Mexico about the state of medical specialists that allow the design of policies for training and use of human resources for health. Objective: To analyze the current state of medical specialists in Mexico: number, distribution (geographical, by gender and specialty), certification validity (by gender and specialty) and the rate of specialists per 100000 population. Method: Cross-sectional, descriptive study. Multiple sources available in Mexico were consulted: councils, associations, schools, hospital institutions, universities, and others. Results: A total of 147,910 specialists were counted for a total population of 123,518,272 inhabitants; $69 \%$ had current specialty certification. A rate of 119 specialists per 100000 population was obtained; $54.2 \%$ are in Mexico City and in the States of Mexico, Jalisco and Nuevo León. On average, there are 1.7 male specialists per female specialist. Conclusions: The number of specialists is lower than that internationally recommended and insufficient to cover the health needs of the country. Gender distribution is shifting towards a more equitable one. Specialists are mainly grouped in urban areas. This is the first census of specialists based on total verifiable number of physicians, which will allow the design of policies for human resources planning in health.
\end{abstract}

KEY WORDS: Medical specialists. Human resources in health. Certified specialists. Mexico.

Correspondencia:

Diana Patricia Guízar-Sánchez

E-mail: guizar.diana@ comunidad.unam.mx
Fecha de recepción: 06-10-2017

Fecha de aceptación: 25-01-2018

DOI://dx.doi.org/10.24875/GMM.18003770
Gac Med Mex. 2018;154:342-351

Disponible en PubMed

www.gacetamedicademexico.com 


\section{Introducción}

Estadísticas de 2015 de la Organización Mundial de la Salud (OMS) refieren 139 médicos por 100000 habitantes en el mundo. ${ }^{1}$ En América Latina, la Organización Panamericana de la Salud (OPS) sugiere 176 médicos por 100000 habitantes. ${ }^{2}$ En México, de acuerdo con el último censo realizado en 2011 por el Instituto Nacional de Estadística, Geografía e Informática había 157 médicos por 100000 habitantes. ${ }^{3}$ El número de médicos per cápita se ha incrementado de 160 por 100000 habitantes en 2000 a 220 en 2014, ${ }^{4}$ sin embargo, continúa siendo inferior al promedio de los países de la Organización para la Cooperación y Desarrollo Económico (OCDE) de 320. ${ }^{4}$ La OCDE en 2015 reportó en México la existencia de más médicos especialistas (ME) que médicos generales y familiares (MG) (65 versus $35 \%$, respectivamente), ${ }^{4}$ cifra similar a la de otros países de la OCDE como Reino Unido ( $\mathrm{ME}=71.4 \%$ versus $\mathrm{MG}=28.6 \%$ ), pero contrastante con Chile ( $M E=51.8 \%$ versus $M G=48.2 \%$ ) y Francia ( $\mathrm{ME}=49.8 \%$ versus $\mathrm{MG}=50.2 \%$ ).

La Comisión Interinstitucional para la Formación de Recursos Humanos para la Salud (CIFRHS), a través del Comité de Posgrado, es la instancia coordinadora del Examen Nacional para Aspirantes a las Residencias Médicas (ENARM). En 2016 compitieron 38077 médicos generales por las 7805 plazas disponibles para iniciar los estudios de especialización. ${ }^{5}$ El Sistema Nacional de Salud cuenta con 81 cursos de especialidad, ${ }^{5}$ de los cuales 78 son ofertados por la Universidad Nacional Autónoma de México (UNAM). ${ }^{6}$

De acuerdo con Zurn et al., ${ }^{7}$ en el sistema de salud mexicano se evidencian cinco tipos de inequidades: geográfica, de género, en la relación de médicos generales y especialistas, en la atención pública y privada, así como de institucionales y servicios.

Hasta donde abarca esta revisión, no hay datos recientes en México sobre el estado actual de los ME para diseñar e impulsar nuevas políticas de formación y empleo de los recursos humanos para la salud. Únicamente, existe un estudio de Heinze et al., ${ }^{8}$ donde se plantean retos en el número, distribución (geográfica y por sexo) y vigencia de la certificación de solo una especialidad médica (psiquiatría).

\section{Objetivo}

Conocer el número total de ME en México, su distribución (geográfica, por sexo y especialidad), el porcentaje de estos con certificación vigente (por sexo y especialidad); estimar la tasa de especialistas por 100000 habitantes (si es acorde con los requerimientos en salud) y realizar una comparación con estándares internacionales, para con ello realizar de forma indirecta una evaluación del estado actual de los especialistas en México.

\section{Método}

Se efectuó un estudio transversal descriptivo por medio de la consulta de registros de la Dirección General de Profesiones de la Secretaría de Educación Pública, los registros de los diferentes consejos pertenecientes al Comité Normativo Nacional de Consejos de Especialidades Médicas (CONACEM), las bases de datos de los diferentes colegios, sociedades o asociaciones de cada especialidad, los registros de los institutos y hospitales con plazas para ejercer 0 realizar las distintas especialidades médicas (Instituto Mexicano del Seguro Social, Instituto de Salubridad y Seguridad Social para los Trabajadores del Estado, Petróleos Mexicanos y Secretaría de Salud) y los directorios de los seguros médicos. Se tomaron en cuenta dichas fuentes por ser consideradas las más confiables para la obtención de datos; la tasa de respuesta fue superior a $90 \%$. La base de datos fue revisada, corregida y validada. Además del número total de ME, se obtuvo su distribución por sexo y se calculó el porcentaje de especialistas certificados.

Con los datos del Consejo Nacional de Población ${ }^{9}$ se determinó la tasa de especialistas por cada 100000 habitantes, tanto en el país como por entidad federativa. Se determinaron aquellas en las que la cifra de especialistas estaba por debajo del promedio recomendado por organismos internacionales.

Esta investigación contó con la aprobación del Comité de Investigación y Ética de la Facultad de Medicina de la UNAM, presentándose los resultados acordes a las 78 especialidades ofertadas en esa institución.

\section{Resultados}

En 2017, en México 147910 ME ejercían su profesión (37.4 \% mujeres y $62.6 \%$ hombres). En la Tabla 1 se muestra un comparativo con otros países de la OCDE. ${ }^{4}$ La relación por sexo fue de 1.7 hombres por cada mujer, la cual varió dependiendo de la especialidad (Tabla 2). En promedio, 69 \% contaba con certificación vigente por el consejo médico de la 
Tabla 1. Médicos especialistas en cuatro países de la Organización para la Cooperación y Desarrollo Económico

\begin{tabular}{lccc}
\hline País & $\begin{array}{c}\text { Población } \\
(\mathbf{n})\end{array}$ & $\begin{array}{c}\text { Total de } \\
\text { especialistas } \\
(\mathbf{n})\end{array}$ & $\begin{array}{c}\text { Especialistas/100 000 } \\
\text { habitantes } \\
\text { (n) }\end{array}$ \\
\hline $\begin{array}{l}\text { Reino } \\
\text { Unido }\end{array}$ & 64956716 & 130563 & 201 \\
Francia & 66357458 & 120107 & 181 \\
México & 123518272 & 147910 & 119 \\
Chile & 18121818 & 19934 & 110 \\
\hline
\end{tabular}

Creado a partir de la información disponible: http://stats.oecd.org/lndex.aspx? DataSetCode=HEALTH_REAC\#.4

especialidad $(73.5 \%$ mujeres versus $64.5 \%$ hombres) al momento del estudio. Urología ginecológica (97.5\%) y biología de la reproducción (97.4\%) fueron las especialidades con mayor porcentaje de ME con certificación vigente, mientras que medicina del trabajo $(20.2 \%)$ y neuropatología $(9.5 \%)$ fueron las especialidades con menor porcentaje (Tabla 3).

Respecto a la distribución geográfica de $\mathrm{ME}$, en la Ciudad de México, Estado de México, Jalisco y Nuevo León se concentró $54.2 \%$ de los ME; el restante $45.8 \%$ se encontró distribuido en las otras 28 entidades federativas. De las 32 entidades federativas de la República Mexicana, la Ciudad de México fue la que contó con mayor tasa de ME (505.7/100 000 habitantes), seguida por Nuevo León (185.3/100 000 habitantes) y Jalisco (172/100 000 habitantes) (Figura 1). Las tasas más bajas las obtuvieron Chiapas (35.9/100 000 habitantes), Guerrero (39.2/100 000 habitantes) y Tlaxcala (51.7/100 000 habitantes).

En la Tabla 4 se muestra la estimación de la tasa (por 100000 habitantes) a nivel nacional de 30 especialidades médicas con mayor número de profesionales, donde se observó importante contraste con especialidades como neonatología, con 76.7 (ME/100 000 habitantes), en tanto que otras como nefrología tienen menos de uno (ME/100 000 habitantes).

\section{Discusión}

Actualmente, la demanda de especialistas médicos es un tema de constante discusión y debate. Se habla de brechas que corresponden a la distancia entre lo que se necesita y lo que se oferta. La generación de recursos humanos para la salud en cantidad adecuada y con las características apropiadas es una de las funciones esenciales de los sistemas de salud. Con el presente trabajo se identifican los siguientes retos:

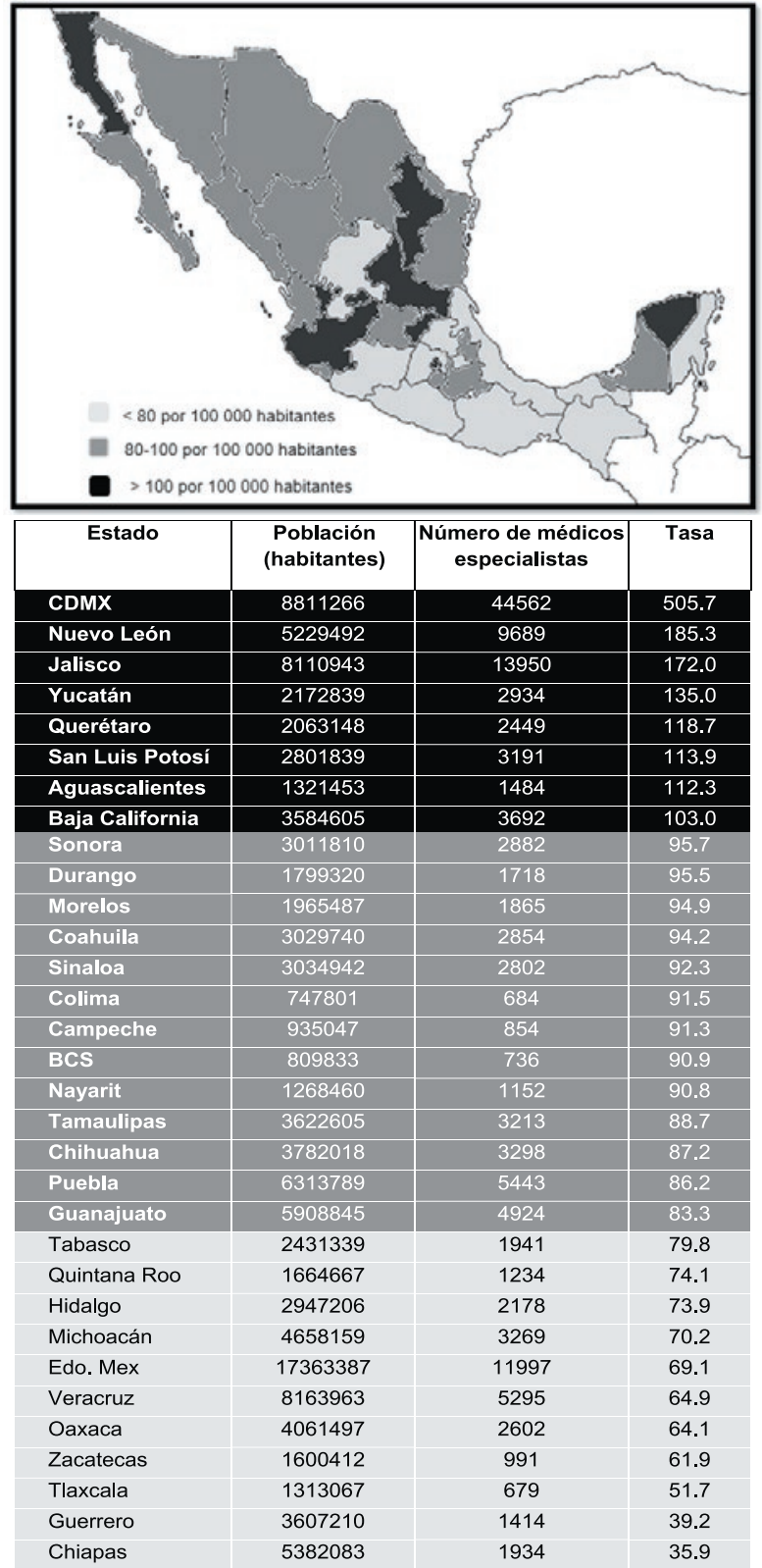

Figura 1. Distribución de ME por entidad federativa. Tasa por 100000 habitantes.

- Número de ME no acorde con la migración, envejecimiento poblacional y la transición epidemiológica de las enfermedades crónico-degenerativas.

- Distribución geográfica inequitativa de especialistas en el país.

- Especialidades con menos de $50 \%$ de médicos con certificación vigente. Aun cuando desde el 2011 es un requisito para la expedición de la cédula profesional de especialista por las autoridades de la Secretaria de Educación Pública (artículo 81 de la Ley General de Salud) ${ }^{11}$ y el 
Tabla 2. Especialidades de mayor contraste en la relación hombre/mujer

\begin{tabular}{lclc}
\hline Especialidades con mayor número de hombres & Relación H:M & Especialidades con mayor número de mujeres & Relación M:H \\
\hline Urología & $45: 1$ & Dermatología pediátrica & $4: 1$ \\
Neurocirugía & $18: 1$ & Audiología, otoneurología y foniatría & $3: 1$ \\
Ortopedia y traumatología & $14: 1$ & Hematología pediátrica & $3: 1$ \\
Cirugía oncológica adultos & $10: 1$ & Endocrinología pediátrica & $2: 1$ \\
Cirugía cardiotorácica & $10: 1$ & Dermatología & $2: 1$ \\
\hline
\end{tabular}

Tabla 3. Total hombres y mujeres especialistas con certificación vigente

\begin{tabular}{|c|c|c|c|c|c|c|}
\hline Especialidad & $\begin{array}{l}\text { Especialistas } \\
\text { hombres } \\
\text { (n) }\end{array}$ & $\begin{array}{c}\text { Hombres con } \\
\text { certificación } \\
\text { vigente (\%) }\end{array}$ & $\begin{array}{l}\text { Especialistas } \\
\text { mujeres } \\
\text { (n) }\end{array}$ & $\begin{array}{l}\text { Mujeres con } \\
\text { certificación } \\
\text { vigente (\%) }\end{array}$ & $\begin{array}{c}\text { Total } \\
\text { especialistas } \\
\text { (n) }\end{array}$ & $\begin{array}{c}\text { Especialistas } \\
\text { con certificación } \\
\text { vigente }(\%)\end{array}$ \\
\hline $\begin{array}{l}\text { Alergia e inmunología } \\
\text { (adultos y pediátrica) }\end{array}$ & 633 & 52.7 & 482 & 54.5 & 1115 & 53.6 \\
\hline Anatomía patológica & 779 & 62.2 & 732 & 57.1 & 1511 & 59.7 \\
\hline $\begin{array}{l}\text { Anestesiología (adultos, pediátrica y } \\
\text { neuroanestesiología) }\end{array}$ & 7889 & 43.9 & 6565 & 56.4 & 14454 & 50.4 \\
\hline Angiología y cirugía vascular & 500 & 49.8 & 106 & 80.1 & 606 & 55.1 \\
\hline Audiología, otoneurología y foniatría & 151 & 60.2 & 464 & 74.1 & 615 & 70.7 \\
\hline Biología de la reproducción humana & 151 & 96.6 & 86 & 98.8 & 237 & 97.4 \\
\hline Cardiología (adultos y pediátrica) & 2473 & 70.25 & 519 & 73.1 & 2992 & 70.7 \\
\hline $\begin{array}{l}\text { Cirugía cardiotorácica (adultos y } \\
\text { pediátrica) }\end{array}$ & 394 & 79.4 & 40 & 90.5 & 434 & 80.8 \\
\hline Cirugía general & 9714 & 34.3 & 1268 & 47.4 & 10982 & 35.8 \\
\hline Cirugía pediátrica & 874 & 65.8 & 166 & 84.9 & 1040 & 68.8 \\
\hline Cirugía oncológica & 794 & 95.4 & 82 & 89.9 & 876 & 94.8 \\
\hline Cirugía plástica y reconstructiva & 1550 & 86.3 & 266 & 85.7 & 1816 & 86.4 \\
\hline Coloproctología & 397 & 71.8 & 64 & 68.8 & 461 & 71.3 \\
\hline $\begin{array}{l}\text { Dermatología (adultos, pediátrica y } \\
\text { dermatopatología) }\end{array}$ & 620 & 60.6 & 1164 & 90.6 & 1784 & 82.1 \\
\hline Endocrinología (adultos y pediátrica) & 675 & 57.7 & 644 & 61.9 & 1319 & 61 \\
\hline Epidemiología & 430 & 58.9 & 259 & 93.1 & 689 & 75.9 \\
\hline $\begin{array}{l}\text { Gastroenterología } \\
\text { (adultos y pediátrica) }\end{array}$ & 827 & 79.8 & 458 & 72.2 & 1285 & 74.3 \\
\hline Genética médica & 107 & 71 & 181 & 91.7 & 288 & 84 \\
\hline Geriatría & 237 & 95.7 & 185 & 96.2 & 422 & 95.9 \\
\hline Ginecología oncológica & 91 & 98 & 60 & 95 & 151 & 97.3 \\
\hline Ginecología y obstetricia & 9654 & 36 & 6035 & 50.6 & 15689 & 41.6 \\
\hline Hematología (adultos y pediátrica) & 310 & 95.3 & 350 & 93.1 & 660 & 90.9 \\
\hline Imagenología diagnóstica-terapéutica & 2282 & 92.1 & 1537 & 89.5 & 3819 & 91 \\
\hline Infectología & 391 & 49.6 & 204 & 75 & 595 & 58.3 \\
\hline
\end{tabular}


Gaceta Médica de México. 2018;154

Tabla 3. Total hombres y mujeres especialistas con certificación vigente (Continuación)

\begin{tabular}{|c|c|c|c|c|c|c|}
\hline Especialidad & $\begin{array}{l}\text { Especialistas } \\
\text { hombres } \\
\text { (n) }\end{array}$ & $\begin{array}{l}\text { Hombres con } \\
\text { certificación } \\
\text { vigente }(\%)\end{array}$ & $\begin{array}{l}\text { Especialistas } \\
\text { mujeres } \\
\text { (n) }\end{array}$ & $\begin{array}{l}\text { Mujeres con } \\
\text { certificación } \\
\text { vigente (\%) }\end{array}$ & $\begin{array}{l}\text { Total } \\
\text { especialistas } \\
\text { (n) }\end{array}$ & $\begin{array}{c}\text { Especialistas } \\
\text { con certificación } \\
\text { vigente }(\%)\end{array}$ \\
\hline $\begin{array}{l}\text { Medicina crítica (adultos y } \\
\text { pediátrica) }\end{array}$ & 1285 & 44.1 & 644 & 52.4 & 1929 & 47.4 \\
\hline $\begin{array}{l}\text { Medicina de la actividad física y } \\
\text { deportiva }\end{array}$ & 115 & 93 & 47 & 89.3 & 162 & 91.9 \\
\hline Medicina familiar & 7831 & 27.6 & 9064 & 44.5 & 16895 & 36.7 \\
\hline Medicina interna & 7123 & 29.8 & 2778 & 39.8 & 9901 & 32.7 \\
\hline Medicina de rehabilitación & 680 & 46 & 1156 & 70.3 & 1836 & 61.3 \\
\hline $\begin{array}{l}\text { Medicina de urgencias (adultos y } \\
\text { pediátrica) }\end{array}$ & 1387 & 72.7 & 1186 & 69.2 & 2573 & 73.5 \\
\hline Medicina legal & 201 & 74.1 & 126 & 75.4 & 327 & 74.6 \\
\hline Medicina materno fetal & 132 & 51.5 & 119 & 89 & 251 & 69.3 \\
\hline Medicina nuclear & 142 & 80.9 & 79 & 84.8 & 221 & 82.3 \\
\hline Medicina del trabajo & 833 & 18.5 & 453 & 23.4 & 1286 & 20.2 \\
\hline Nefrología (adultos y pediátrica) & 694 & 89.5 & 423 & 97.5 & 1117 & 93.9 \\
\hline Neonatología & 743 & 60 & 973 & 67.4 & 1716 & 64.2 \\
\hline Neumología (adultos y pediátrica) & 720 & 76.5 & 351 & 96.1 & 1071 & 83 \\
\hline $\begin{array}{l}\text { Neurocirugía } \\
\text { (adultos, pediátrica y terapia } \\
\text { endovascular neurológica) }\end{array}$ & 1076 & 71.9 & 70 & 89.9 & 1146 & 73.3 \\
\hline Neurofisiología clínica & 83 & 72.3 & 52 & 90.3 & 135 & 79.2 \\
\hline Neurología (adultos y pediátrica) & 906 & 49.5 & 439 & 68.3 & 1345 & 54.8 \\
\hline Neuropatología & 13 & 7.7 & 8 & 12.5 & 21 & 9.5 \\
\hline Neurorradiología & 75 & 48 & 28 & 32.1 & 103 & 43.7 \\
\hline Nutrición clínica & 49 & & 74 & & 123 & \\
\hline $\begin{array}{l}\text { Oftalmología y oftalmología } \\
\text { neurológica }\end{array}$ & 2649 & 61 & 1782 & 71.5 & 4431 & 65.2 \\
\hline $\begin{array}{l}\text { ORL y cirugía de cabeza y } \\
\text { cuello (adultos, pediátrica y } \\
\text { neurootología) }\end{array}$ & 1675 & 82.7 & 1064 & 83.8 & 2739 & 83.1 \\
\hline $\begin{array}{l}\text { Oncología médica } \\
\text { (adultos y pediátrica) }\end{array}$ & 331 & 87.2 & 279 & 88.4 & 610 & 86.7 \\
\hline Ortopedia y traumatología & 6775 & 48.3 & 481 & 53 & 7256 & 48.5 \\
\hline $\begin{array}{l}\text { Patología clínica } \\
\text { (adultos y pediátrica) }\end{array}$ & 445 & 46.9 & 313 & 61.9 & 758 & 53.2 \\
\hline Pediatría & 9499 & 47.6 & 9272 & 64.1 & 18771 & 55.8 \\
\hline Psiquiatría (adultos y pediátrica) & 2836 & 35.2 & 1593 & 38.5 & 4429 & 35.1 \\
\hline Radiooncología & 167 & 94.6 & 71 & 98.5 & 238 & 95.7 \\
\hline Reumatología (adultos y pediátrica) & 521 & 56.4 & 379 & 73.3 & 900 & 78.4 \\
\hline Urología & 1663 & 68.2 & 37 & 81 & 1700 & 68.5 \\
\hline Urología ginecológica & 44 & 95.4 & 36 & 97.2 & 80 & 97.5 \\
\hline
\end{tabular}


Tabla 4. Médicos especialistas en México por cada 100000 habitantes ${ }^{12}$

\begin{tabular}{|c|c|}
\hline Especialidad & Tasa por 100000 \\
\hline Neonatología* & 76.71 \\
\hline Pediatría** & 49.55 \\
\hline Ginecología y obstetricia $^{\dagger}$ & 30.33 \\
\hline Medicina familiar & 14.08 \\
\hline Anestesiología & 11.51 \\
\hline Cirugía general & 8.89 \\
\hline Medicina interna & 8.02 \\
\hline Ortopedia y traumatología & 5.87 \\
\hline Oftalmología & 3.56 \\
\hline Psiquiatría & 3.4 \\
\hline Geriatría $^{\dagger \dagger}$ & 3.25 \\
\hline Imagenología diagnóstica y terapéutica & 3.09 \\
\hline Cirugía pediátrica* & 2.75 \\
\hline Cardiología & 2.16 \\
\hline Otorrinolaringología y cirugía de cabeza y cuello & 2.08 \\
\hline Medicina de rehabilitación & 1.49 \\
\hline Cirugía plástica y reconstructiva & 1.47 \\
\hline Urología & 1.38 \\
\hline Medicina de urgencias & 1.25 \\
\hline Endocrinología & 0.80 \\
\hline Medicina materno-fetal $\left.\right|^{\ddagger}$ & 0.73 \\
\hline Nefrología & 0.72 \\
\hline Cirugía oncológica & 0.71 \\
\hline Neumología & 0.69 \\
\hline Biología de la reproducción humana ${ }^{\ddagger}$ & 0.69 \\
\hline Oncología pediátrica* & 0.60 \\
\hline Epidemiología & 0.56 \\
\hline Infectología & 0.48 \\
\hline Oncología médica & 0.31 \\
\hline Ginecología oncológica ${ }^{\dagger}$ & 0.29 \\
\hline
\end{tabular}

CONACEM establece la renovación de vigencia cada cinco años.

Respecto al número total de $\mathrm{ME}$, resulta necesario revisar de manera integral el proceso de planificación de su formación.
En cuanto a la formación médica general debe señalarse que en México se gradúan anualmente más médicos que en el promedio de los países de la OCDE (12 versus 10 por cada 100000 habitantes, respectivamente). ${ }^{4}$ Es uno de los países con mayor número de escuelas de medicina:12 según el Reconocimiento de Validez Oficial de Estudios ${ }^{13}$ de la Secretaría de Educación Pública actualmente existen 160 escuelas de medicina en el país, de las cuales solo 70 están acreditadas ante el Consejo Mexicano para la Acreditación de la Educación Médica (http://www. comaem.org.mx/), sin que se cuente con una política nacional que regule el volumen de nuevos estudiantes, la calidad de la formación o que dicho aumento responda a una planeación estratégica acorde con las necesidades epidemiológicas y demográficas de las entidades federativas o del país. A su vez, la licenciatura de médico cirujano suele verse como un trámite necesario para ser especialista y no como una opción de ejercicio profesional.

Respecto al número de especialistas, pese al importante egreso de médicos generales en México, la tasa de especialistas es menor al promedio de otros países (178 versus 119 por cada 100000 habitantes), ${ }^{4}$ superior a la de países como Chile (119) pero inferior a la de Reino Unido (201) y Francia (181). Sería importante valorar las diferencias en la planificación de especialistas:

- Reino Unido: Planificación descentralizada en los niveles locales y regionales a través del Consejo Nacional para el Desarrollo de Personal de Salud, que analiza las demandas y necesidades de personal especializado y la formación de especialistas, de una manera congruente con el enfoque que prevalece en la Unión Europea. ${ }^{14}$

- Francia: El Ministerio de Educación Superior y el Ministerio de Salud determinan la lista de especialidades disponibles, dependiendo de las necesidades de salud y de investigación en salud; el gobierno de Francia ajusta el número de disposiciones propuestas por dichos ministerios para cada especialidad en conjunto con los diferentes comités y estancias de la Facultad de Medicina. ${ }^{15}$

- México: Las instituciones de salud envían el número de puestos de residencia disponibles para cada especialidad a la CIFRHS anualmente, que se encarga de la planificación del ENARM. Después de haber aprobado, los residentes se asignan a las posiciones disponibles de acuerdo con su puntuación. ${ }^{14}$ 
- Chile: Se desarrolló una metodología que considera la fuerza de trabajo disponible, estimando con esto el número de especialistas que se requieren. El responsable de la planificación es el Ministerio de Salud en conjunto con las universidades del país que cuenten con programas de formación de ME. ${ }^{14}$

Es importante analizar el número de especialidades reconocidas en un país, ya que mientras más especialidades o nomenclaturas de especialidades existan, mayor será su déficit. Una revisión de los programas de especialidades médico-quirúrgicas en América Latina refiere gran diferencia en el número de especialidades reconocidas, que oscila entre seis (EI Salvador) y 82 (Colombia). ${ }^{16}$ Por su parte, la Asociación de Escuelas de Medicina de Estados Unidos (https:// www.aamc.org/), en su informe de aspirantes a especialidad, reporta 45 especialidades, cifra similar a la de Costa Rica y Argentina, ${ }^{16}$ mientras que el Ministerio de Sanidad, Servicios Sociales e Igualdad de España y la Unión Europea de Médicos Especialistas reportan 50 especialidades médicas (https://www. uems.eu/).

No solo es importante revisar el número total de $\mathrm{ME}$, sino que el incremento de los mismos ha sido inercial y no guarda relación con el crecimiento poblacional ni las modificaciones del perfil epidemiológico, lo que impide una adecuada planeación de la cobertura de la atención médica. ${ }^{17}$

En México, en los últimos 30 años se han incrementado las enfermedades no transmisibles: diabetes, enfermedades respiratorias crónicas, enfermedades cardiovasculares, insuficiencia renal crónica, ${ }^{17}$ trastornos hepáticos, musculoesqueléticos, enfermedades de salud mental (depresión) y la discapacidad por problemas lumbares. ${ }^{18}$

En México existe un mayor número de pediatras (49/100 000 niños) y ginecólogos (30/100 000 mujeres), pese a que la tasa de fertilidad se ha reducido de forma importante (en los últimos 50 años de 6.7 a 2.1)..$^{19}$ Respecto a los psiquiatras, aun cuando la enfermedad mental representa $13 \%$ de la carga global de enfermedad, ${ }^{20}$ en el país solo existen 4195 psiquiatras, es decir, 3.4 por cada 100000 habitantes (cifra inferior a la recomendación de 5/100 000 de la OMS). ${ }^{20}$ Acorde con los datos obtenidos en este estudio, se identifica una aparente falta de médicos en algunas especialidades:

- Se contabilizaron 9901 internistas (8/100 000 habitantes) y 2671 cardiólogos (2/100 000 habitantes) para la atención de las enfermedades cardiovasculares, segundo problema de salud que tiene el país.

- Se dispone de 895 nefrólogos, pese al incremento que han tenido los problemas renales.

- No obstante que la enfermedad pulmonar obstructiva crónica y el cáncer pulmonar exhiben tasas crecientes de mortalidad y que la suma de ellas rebasa significativamente a la cardiopatía isquémica como causa de muerte global ( $16.7 \%$ versus $12.8 \%$ ), ${ }^{21-23}$ se encontró un neumólogo por 200000 habitantes, cifra inferior a la recomendada por la OMS (3.5 a 4 neumólogos/100 000 habitantes).

- En México, las personas adultas mayores constituyen un grupo que registra un crecimiento acelerado (para 2050 representará $27 \%$ de la población)..$^{17}$ Los estándares internacionales recomiendan un geriatra por 50000 habitantes (http://www.bgs.org.uk/) o un geriatra por 2000 adultos mayores (http://www.americangeriatrics. org/), cifra 15 veces superior a la encontrada en este estudio de tres geriatras por cada 100000 adultos mayores.

Frente a este escenario se requiere (antes de aumentar el número de especialistas, que resultaría costoso y no resolvería el problema de fondo), en primer lugar, implementar la detección oportuna de enfermedades crónico-degenerativas, evitando así complicaciones de alto costo como la insuficiencia renal o las enfermedades cardiovasculares. Como podemos observar, las enfermedades no transmisibles comparten cuatro factores de riesgo: consumo de tabaco, consumo excesivo de alcohol, dieta no saludable e inactividad física; por lo que invertir en temas de promoción, minimización de la exposición a factores de riesgo, maximización de exposición a factores protectores, prevención y detección oportuna, sentará las bases para fortalecer el cambio hacia un modelo de atención primaria de salud, donde los médicos familiares y generales desempeñan el papel más relevante.

Las instituciones de salud necesitan urgentemente regular la productividad, mejorar la calidad de la atención y el equilibrio entre el número de especialistas versus médicos familiares (acorde con el estudio 14/100 000 habitantes). Datos de la Secretaría de Salud muestran que los indicadores de productividad de los últimos 10 años son sumamente bajos entre los ME (dos consultas diarias por médico), pero comparativamente altos entre médicos generales y familiares (13 consultas diarias por médico). ${ }^{24}$ 
Se requiere una adecuada comunicación entre las instituciones educativas y las de salud, ya que actualmente los ME atienden a la población aplicando el método clínico-curativo, estableciendo el diagnóstico y tratamiento adecuado a cada caso, pero el resto de las acciones como la educación, promoción de un estilo de vida saludable, prevención, control y seguimiento del paciente son ejecutadas por el personal de salud formado en áreas técnicas de enfermería, nutrición, psicología, entre otros. Por lo tanto, resulta crucial la transformación de un modelo netamente curativo a uno preventivo-curativo. A su vez, replantear para algunas especialidades, como pediatría y ginecología y obstetricia, una reducción en el número de plazas laborales e incrementar las de médicos familiares y generales capacitados. Finalmente, un reacomodo o un incremento importante de plazas en especialidades necesarias para atender la patología emergente, para lo que ayudaría el cambio de los requisitos de entrada y duración de algunas especialidades.

Acorde con lo reportado en 2016 por la CIFRHS, $75 \%$ de los residentes recientemente ingresados están distribuidos en seis especialidades médicas principales: medicina familiar $(21.8 \%)$, medicina interna (13.5\%), anestesiología (10.8\%), pediatría (10.8\%) ginecología y obstetricia $(9.9 \%)$ y cirugía $(9.1 \%)$ (http://www.cifrhs.salud.gob.mx/). Actualmente, dentro de la plantilla escolar de la UNAM dichas especialidades representan $47 \%$ de los alumnos. ${ }^{6}$ El cambio de vía de entrada, de indirecta a directa, anteriormente ha solucionado el déficit del número de aspirantes en algunas especialidades médicas y, por el contrario, al convertirlas de entrada directa a indirecta ha desalentado la solicitud de aspirantes. ${ }^{25}$

La distribución geográfica de ME es poco equitativa, similar a lo que sucede en otros países, ${ }^{25-27} \mathrm{con}$ una clara centralización y desigualdad en la asignación de recursos para la formación de especialistas. Lo que podría estar relacionado con los siguientes aspectos:

- Que 9626 de 26789 alumnos de especialidad y 78 de 81 programas corresponden a la Facultad de Medicina de la UNAM. ${ }^{6}$ Cuya plantilla escolar de 2016 se concentró en la Ciudad de México (8644 de 9626 alumnos). Por lo tanto, se requiere una adecuada planeación de plazas de residencias médicas, que no necesariamente implica incrementar las plazas en las sedes existentes, sino abrir nuevas sedes de especialidad en las diferentes entidades federativas.
- La falta de ofertas de trabajo, reconocimiento y estímulos a especialistas egresados para lograr su retención en instituciones públicas de ciudades de pequeña y mediana población.

- La concentración del desarrollo económico y social, es decir, en algunas entidades federativas del país se tiene un menor ingreso per cápita, escaso o nulo acceso a educación básica de calidad (pensando en las familias de los ME egresados), poca disponibilidad de servicios básicos (alimentación, vivienda, etcétera), carencia de infraestructura física para el ejercicio adecuado de la profesión y escasa o nula posibilidad de ejercer la práctica privada en conjunción con el ejercicio público de la profesión. Por lo tanto, se debe buscar crear condiciones para hacer más atractivo a los ME el ejercicio en zonas semiurbanas y rurales.

- Entidades federativas con altos índices de violencia con escasa o nula garantía de seguridad. De acuerdo con la Encuesta Médica Nacional de Riesgo, la Ciudad de México, Chihuahua, Coahuila, Hidalgo, Guerrero, Morelos, Nuevo León, Tamaulipas, Oaxaca y Veracruz son las entidades federativas con mayor reporte de violencia. ${ }^{28}$

Otro aspecto importante es la feminización del trabajo médico, que constituye un fenómeno global. En varios países de la OCDE la proporción de médicas es casi equivalente a la de médicos ( $46 \%$ en Reino Unido y $43 \%$ en Francia). ${ }^{4}$ Levinson y Lurie ${ }^{29}$ proponen que las mujeres y los hombres practican la medicina de manera diferente; las mujeres pueden mejorar la relación médico-paciente y promover más trabajo en equipo debido a características atribuidas al sexo femenino como la compasión, la empatía, la capacidad de respuesta a las necesidades del paciente y las habilidades de comunicación. ${ }^{30,31}$ Por otro lado, hay estudios que refieren que algunos pacientes prefieren ser atendidos por un hombre en servicios de atención primaria, hecho que generaría problemas en la búsqueda de un diagnóstico y tratamiento oportuno. ${ }^{32-34} \mathrm{~A}$ nivel internacional se tiene una visión más ambivalente de las implicaciones del creciente número de mujeres en medicina. Por un lado, están dispuestas a trabajar en posiciones menos bien compensadas y practicar en especialidades de atención primaria, pero, por otro lado, prefieren ejercer en zonas urbanas, probablemente por la necesidad de más apoyo para combinar la maternidad con la carrera, evitar el agotamiento y ganar representación en el liderazgo y los académicos. ${ }^{35}$ 
Respecto a la distribución por sexo en México, se sabe que la población de mujeres que ingresan a estudiar medicina y que posteriormente realizan una residencia médica se ha incrementado de manera gradual, pero con diferencias en la relación hombre: mujer dependiendo de la especialidad médica; se ha sugerido que dichas diferencias se deben a la percepción del estilo de vida de la especialidad, observándose cierta tendencia en los hombres a la elección de aquellas con un estilo de vida no controlable:36 medicina interna, ginecología y obstetricia, ortopedia, cirugía general y urología; y las mujeres a aquellas con estilo de vida controlable: anestesiología, dermatología, neurología, patología, psiquiatría y radiología.

En México no todos los médicos que egresan de su formación como residentes se certifican, y quienes han estado certificados no suelen recertificarse. Especialidades como urología ginecológica y biología de la reproducción tienen casi $100 \%$ de ME con certificación vigente; mientras que en cirugía general, medicina interna, psiquiatría y neuropatología menos de $40 \%$. Dicha variación puede ser secundaria a diversos factores:

- En medicina interna y cirugía general puede ser secundaria a que los especialistas que optaron por una segunda especialidad o subespecialidad y ya no se recertificaron como internistas o cirujanos generales.

- En las especialidades con gran número de especialistas con certificación vigente (ginecología oncológica, biología de la reproducción y urología ginecológica) probablemente por el grado de sobreespecialización de las mismas.

- A las variaciones en la promoción que se tiene en relación con la capacitación y actualización de los especialistas por cada uno de los consejos médicos de la especialidad.

Entre las fortalezas del estudio podemos mencionar que se trata del primer censo de especialistas que se realiza con base en el número total comprobable de médicos y no un conteo realizado tomando como referencia las plazas ocupadas, lo cual genera datos duplicados. ${ }^{14}$ Es el primer estudio en plantear una distribución por género, especialidad, estado de la república y vigencia de la certificación de más de $90 \%$ de las especialidades médicas disponibles en el país.

Las limitaciones radican en la disponibilidad de los datos: solo los especialistas de los que se tenía información en alguna de las bases de datos pudieron ser contados y localizados.

Antes de incrementar el número de ME se debe realizar otras acciones:

- Garantizar que las instituciones de educación superior egresen al personal que la población y las instituciones de salud requieren, tanto en cantidad como en enfoque educativo (pasar de uno centrado en la enfermedad a uno preventivo).

- Reforzar la importancia del médico general, ya que en contraste con los ME, en la Ciudad de México se tiene el menor porcentaje de médicos en atención primaria. ${ }^{37}$

- Evitar la subutilización de los médicos, garantizando un espacio en el mercado laboral a los médicos generalistas (familiares y generales) y especialistas con igualdad de oportunidades en las diferentes entidades federativas.

- Evitar la sobreespecialización médica mediante la regulación en los programas educativos (enfoque formativo, requisitos formativos de ingreso $y$ duración de cada especialidad médica).

- Limitar la creación de nuevas especialidades médicas, que fragmenten aún más el conocimiento, lo que va en contra de la tendencia mundial que apunta a un modelo integrador y de prevención primaria (que a la larga resulta menos costoso que el actual).

- Tomar en cuenta la feminización del trabajo médico, debido a que las mujeres requieren apoyo y tiempo para combinar el ejercicio de la profesión con el cuidado de los hijos.

Por lo tanto, se requiere articular la formación médica general -cambio a un paradigma preventivo, reducir la cantidad de egresados y crear organismos que regulen la calidad académica de los mismos el mercado laboral (tanto de ME, como generales y familiares, en todos los niveles de atención y entidades federativas) y el patrón epidemiológico mixto enfermedades crónico-degenerativas junto con enfermedades de rezago social y pobreza-, con el fin de llevar a cabo una planeación estratégica de la especialización médica; cuyo objetivo final sea la distribución suficiente y equilibrada de los recursos humanos para la salud.

\section{Agradecimientos}

Al doctor Onofre Muñoz-Hernández, presidente del CONACEM. 


\section{Bibliografía}

1. World Health Statistics 2015. Ginebra: World Health Organization; 2015

2. Situación de salud en las Américas, Indicadores Básicos 2015. USA Organización Panamericana de la Salud/Organización Mundial de la Salud; 2015.

3. Instituto Nacional de Estadística y Geografía. [Sitio web]. Sociedad. Cuadro resumen indicadores sociales. [Consultado 2017 Abr 16]. Disponible en: http://www3.inegi.org.mx/sistemas/temas/default.aspx?s=est\&c=21702

4. Organization for Economic Co-operation and Development. [Sitio web] Health care resources. [Consultado 2017 Jun 8]. Disponible en: http:// stats.oecd.org/Index.aspx?DataSetCode=HEALTH_REAC\#.Data

5. Comisión Interinstitucional para la Formación de Recursos Humanos para la Salud. [Sitio web]. XL Examen Nacional para Aspirantes a Residencias Médicas. [Consultado 2017 Jun 7]. Disponible en: http://www. cifrhs.salud.gob.mx/site1/enarm/2016.html

6. Universidad Nacional Autónoma de México. Especializaciones 2018 México: Facultad de Medicina; 2017. [Consultado 2017 Jun 8]. Disponible en: https://escolares.facmed.unam.mx/doc/oficinaVirtual/alumnos/ posgrado/instructivos/folletoBienvenidos2017.pdf

7. Zurn P, Dal Poz MR, Stilwell B, Adams O. Imbalance in the health workforce. Hum Resour Health. 2004;2:13.

8. Heinze G, Chapa GC, Carmona-Huerta J. Los especialistas en psiquiatría en México; año 2016. Salud Mental. 2016;39:69-76.

9. Proyecciones de la Población 2010-2050. México: Consejo Nacional de Población; 2017.

10. Encuesta Nacional de Salud y Nutrición 2012. Resultados nacionales. México: Instituto Nacional de Salud Pública; 2012.

11. Espinoza-De-Los-Reyes SVM. Participación de la Academia Nacional de Medicina de México en la acreditación de las especialidades y la certificación de médicos especialistas. Gac Med Mex. 2014;150:227-234

12. Frenk J, Chen L, Bhutta ZA, Cohen J, Crisp N, Evans T, et al. Health professionals for a new century: transforming education to strengthen health systems in an interdependent world. Lancet. 2010;376(9756): 1923-1958.

13. Secretaría de Educación Pública. [Sitio web]. Reconocimiento de Validez Oficial. [Consultado 2017 Jun 5]. Disponible en: http://www.sep.gob.mx/ es/sep1/Revoe\#.WhtWZvWcHmQ

14. Fajardo DG, Santacruz VJ, Lavalle MC. La formación de médicos especialistas en México. Documento de postura. México: Academia Nacional de Medicina; 2015

15. Miani C, Hinrichs S, Pitchforth E, Bienkowska-Gibbs T, Disbeschl S, Roland $M$, et al. Best practice: Medical training from an international perspective. Rand Health Q. 2015:5:2.

16. Residencias médicas en América Latina. USA: Organización Panamericana de la Salud/Organización Mundial de la Salud; 2011

17. Secretaría de Salud, Anuario de morbilidad 1984-2016. México: Dirección General de Epidemiología; 2017. [Consultado 2017 May 1]. Disponible en: https://www.gob.mx/salud/acciones-y-programas/anuarios-de-morbilidad-1984-2016
18. Institute for Health Metrics and Evaluation. Data visualizations. [Consultado 2017 Jun 15]. Disponible en: http://www.healthdata.org/results/data-visualizations/

19. The World Bank. Fertility rate, total (births per woman). [Consultado 2017 Jun 19]. Disponible en: http://data.worldbank.org/indicator/SP.DYN. TFRT.IN/

20. Mental health atlas 2014. Ginebra: World Health Organization; 2015.

21. Sandoval-Gutiérrez JL, Sevilla RS, Bautista BB. Pulmonary diseases: First cause of mortality in the world. Chest. 2011;139:1550.

22. Organización Mundial de la Salud. [Sitio web]. ¿Cuál es la enfermedad que causa más muertes en el mundo? [Consultado 2017 Jun 15]. Disponible en: http://www.who.int/features/qa/18/es

23. The World Bank. [Sitio web]. Datos, América Latina y el Caribe. [Consultado 2017 Jun 19]. Disponible en: http://datos.bancomundial.org/region/LAC

24. Nigenda G, Muños JA. Projections of specialist physicians in Mexico: a key element in planning human resources for health. Human Resour Health. 2105;13:79.

25. Sepúlveda CC. Formación de especialistas médicos en Chile. Rev Chil Cir. 2012;64:507.

26. Ministerio de Salud y Protección Social de la República de Colombia Estudio de disponibilidad y distribución de la oferta de médicos especialistas, en servicios de alta y mediana complejidad en Colombia. Informe final. Colombia: Pontificia Universidad Javeriana/Centro de Protección para el Desarrollo; 2013

27. Navarro PP, Contreras SAJ, Junco GMC, Sánchez VP, Sánchez-Cantalejo GC Luque MN. Análisis de la distribución geográfica de médicos especialistas en la República Argentina. Argentina: OPS/OMS: 2015.

28. Rivera G. Tierras violentas sin médicos. Newsweek. 2017:21:8-13.

29. Levinson W, Lurie N. When most doctors are women: what lies ahead? Ann Intern Med. 2004;141:471-474.

30. Eagly AH, Johannesen-Schmidt MC, Van-Engen ML. Transformational, transactional, and laissez-faire leadership styles: a meta-analysis comparing women and men. Psychol Bull. 2003;129:569-591.

31. Roter DL, Hall JA., Aoki Y. Physician gender effects in medical communication: a meta-analytic review. JAMA. 2002;288:756-764.

32. Derose KP, Hays RD, McCaffrey DF, Baker DW. Does physician gender affect satisfaction of men and women visiting the emergency department? J Gen Intern Med. 2001;16:218-226.

33. Franks $P$, Bertakis KD. Physician gender, patient gender, and primary care. J Womens Health (Larchmt). 2003;12:73-80.

34. Pasko T, Smart DR. Physician characteristics and distribution in the U.S. 2004-2005. USA: American Medical Association; 2004.

35. Spenny ML, Ellsbury KE. Perceptions of practice among rural family physicians-is there a gender difference? J Am Board Fam Pract. 2000;13:183-187

36. Bickel J, Ruffin A. Gender-associated differences in matriculating and graduating medical students. Acad Med. 1995;70:552-559.

37. Nigenda G, Alcalde-Rabanal J, González-Robledo LM, Serván-Mori E, García-Saiso S, Lozano R. Eficiencia de los recursos humanos en salud: una aproximación a su análisis en México. Salud Publica Mex. 2016;58:533-542. 